\title{
Influence of strain and oxygen vacancies on the magnetoelectric properties of multiferroic bismuth ferrite
}

\author{
Claude Ederer* and Nicola A. Spaldin \\ Materials Research Laboratory and Materials Department, University of California, Santa Barbara, California, 93106, USA
}

(Received 15 February 2005; published 13 June 2005)

\begin{abstract}
The dependencies on strain and oxygen vacancies of the ferroelectric polarization and the weak ferromagnetic magnetization in the multiferroic material bismuth ferrite, $\mathrm{BiFeO}_{3}$, are investigated using first principles density functional theory calculations. The electric polarization is found to be rather independent of strain, in striking contrast to most conventional perovskite ferroelectrics. It is also not significantly affected by oxygen vacancies, or by the combined presence of strain and oxygen vacancies. The magnetization is also unaffected by strain, however, the incorporation of oxygen vacancies can alter the magnetization slightly, and also leads to the formation of $\mathrm{Fe}^{2+}$. These results are discussed in light of recent experiments on epitaxial films of $\mathrm{BiFeO}_{3}$, which reported a strong thickness dependence of both magnetization and polarization.
\end{abstract}

DOI: 10.1103/PhysRevB.71.224103

PACS number(s): 71.15.Mb, 75.70.Ak, 77.80.-e

\section{INTRODUCTION}

Materials that simultaneously show electric and magnetic order are currently gaining more and more attention. This is partly due to the fact that such multiferroics are promising materials for the design of multifunctional device applications, but also because of the interesting physics found in this class of materials. For example, a strong coupling between ferroelectric and antiferromagnetic domain walls has been found in $\mathrm{YMnO}_{3},{ }^{1}$ in orthorhombic $\mathrm{TbMnO}_{3}$ and $\mathrm{TbMn}_{2} \mathrm{O}_{4}$ the ferroelectric polarization can be reoriented by a magnetic field, 2,3 and ferromagnetic ordering can be "switched on" by an electric field in hexagonal $\mathrm{HoMnO}_{3} \cdot{ }^{4}$

Although magnetoelectric materials have been known for a long time,$^{5}$ recent progress in thin-film growth and other sample preparation techniques contributed considerably to their renaissance. By using techniques such as pulsed laser deposition (PLD), chemical vapor deposition (CVD), or molecular beam epitaxy (MBE), many materials can nowadays be prepared as high quality epitaxial thin films. One advantage of these techniques is the possibility to stabilize otherwise metastable structures or to tune material properties by varying the lattice mismatch between the film and the substrate, thereby introducing epitaxial strain in the thin film material.

Indeed, the effect of strain on the ferroelectric properties of conventional ferroelectric materials is also a topic of current interest. Strain effects can lead to a substantial increase of the spontaneous polarization and Curie temperature, ${ }^{6}$ and even drive paraelectric materials (such as $\mathrm{SrTiO}_{3}$ ) into the ferroelectric phase. ${ }^{7}$ Since the mechanism for ferroelectricity in multiferroic materials is often different from that in conventional perovskite ferroelectrics, ${ }^{8,9}$ the question arises of whether similar strain effects will be observed in multiferroics. Magnetic properties can also be strongly affected by strain, mainly due to large changes in anisotropy. ${ }^{10}$ Strain can also affect the saturation magnetization and Curie temperature. ${ }^{11}$

First principles density functional theory (DFT) calculations (see, e.g., Ref. 12) play a crucial role in studying the influence of strain on ferroelectric properties. ${ }^{13-15}$ Epitaxial strain can be introduced straightforwardly in DFT studies by fixing the lattice parameters in the directions corresponding to the lateral dimension of the substrate and allowing the system to relax in the perpendicular direction. This makes it possible to clearly distinguish between the effect of strain and other influences present in real thin-film samples, such as interface effects or various types of defects. Such information can then be used to optimize the properties of the thin-film material.

In this work we study the influence of strain on the electric polarization and magnetization of multiferroic bismuth ferrite, $\mathrm{BiFeO}_{3}$. Bismuth ferrite crystallizes in a rhombohedrally distorted perovskite structure with space group $R 3 c,{ }^{16}$ where all ions are displaced along the [111] direction relative to the ideal centrosymmetric positions, and the oxygen octahedra surrounding the transition metal cations are rotated around this axis, alternately clockwise and counterclockwise. The magnetic order is essentially $G$-type antiferromagnetic ${ }^{17}$ but in addition, the direction of the magnetic moments in the bulk rotates with a long wavelength of $620 \AA$ (Ref. 18). We have recently shown that if this spiral spin structure is suppressed, the system shows weak ferromagnetism ${ }^{19}$ with the magnetic moments oriented perpendicular to the rhombohedral axis and a slight canting of these magnetic moments resulting in a small macroscopic magnetization. ${ }^{20}$

Ferroelectric hysteresis loops have been measured for $\mathrm{BiFeO}_{3}$ but the experimental determination of an exact value for the spontaneous polarization $P_{S}$ is difficult due to large leakage currents. ${ }^{21}$ Several values are reported in the literature, summarized in Table I of Ref. 22. A first principles calculation of the spontaneous polarization of bulk $\mathrm{BiFeO}_{3}$ results in a value of $P_{s} \sim 95 \mu \mathrm{C} / \mathrm{cm}^{2}$ (Ref. 22); recent measurements for epitaxial films grown on $\mathrm{SrTiO}_{3}$ agree well with this value. ${ }^{23-25}$ These experiments also show a strong dependence of both magnetization and ferroelectric polarization on the film thickness. ${ }^{23}$ One likely explanation for this thickness dependence is the increase in strain with decreasing film thickness; another is a change in the concentration of defects such as oxygen vacancies. In this work we sys- 
tematically examine both possibilities using first principles DFT calculations.

Our main result is that the strain dependence of the ferroelectric polarization in $\mathrm{BiFeO}_{3}$ is rather weak compared with conventional ferroelectric materials and that it cannot explain the variation of the polarization reported for the thin film samples. The same is true for the magnetization, which also shows only weak strain dependence. The weak strain dependence of the polarization is due to a very stable ionic configuration in $\mathrm{BiFeO}_{3}$, which manifests itself in only small changes of the relative ionic positions when the lattice is strained.

The high stability of the ferroelectric configuration also leads to a negligible dependence of the electric polarization on the oxygen vacancy concentration. In contrast, we find that the magnetization of $\mathrm{BiFeO}_{3}$ is affected by the presence of oxygen vacancies but the changes are not very systematic and depend on the precise position of the oxygen vacancy. The presence of oxygen vacancies in all cases leads to the formation of $\mathrm{Fe}^{2+}$ ions, which can be identified unequivocally in the partial densities of states although the actual charge differences between the different $\mathrm{Fe}$ sites are very small.

\section{METHOD}

For this work we use first principles density functional theory (see, e.g., Ref. 12) within the projector augmented wave (PAW) method ${ }^{26}$ as implemented in the Vienna Ab initio Simulation Package (VASP). ${ }^{27,28}$ We include 15 valence electrons for $\mathrm{Bi}\left(5 d^{10} 6 s^{2} 6 p^{3}\right), 14$ for $\mathrm{Fe}\left(3 p^{6} 3 d^{6} 4 s^{2}\right)$, and 6 for each oxygen $\left(2 s^{2} 2 p^{4}\right)$, use an energy cutoff between 450 and $500 \mathrm{eV}$ for the plane wave expansion of the PAWs, a $4 \times 4 \times 4$ Monkhorst Pack grid of $k$ points, ${ }^{29}$ and the tetrahedron method with Blöchl corrections for the Brillouin zone integrations. ${ }^{30}$ For all structures (unless otherwise noted) we relax the ionic positions while keeping the lattice vectors fixed until the Hellman-Feynman forces are less than $10^{-2} \mathrm{eV} / \AA$. For the calculation of the local densities of states at the Fe sites we use a sphere radius of $1.4 \AA$. These values have been found to give good convergence of all quantities under consideration.

To treat exchange and correlation effects we use both the local spin density approximation (LSDA) ${ }^{12}$ and the semiempirical LSDA $+\mathrm{U}$ method $^{31}$ for a better description of the localized transition metal $d$ electrons. We have recently shown that using the LSDA $+\mathrm{U}$ method and a moderate value of $U=3 \mathrm{eV}$ (and $J=1 \mathrm{eV}$ ) leads to a good description of the structural parameters and the ferroelectric polarization in $\mathrm{BiFeO}_{3} .{ }^{22}$ Larger $U$ values shift the $d$ bands further down in energy relative to the oxygen $p$ states but have only a small effect on the structural and ferroelectric properties. $U$ $=3 \mathrm{eV}$ can be regarded as a lower limit of what is required to ensure the insulating character of $\mathrm{BiFeO}_{3}$, and here we exclusively use this value (and $J=1 \mathrm{eV}$ ) in our LSDA+U calculations. We do not claim that these values necessarily would also lead to a good description of spectroscopic quantities such as, e.g., photoemission spectra.

There are two different LSDA+U approaches imple- mented in the VASP code, (i) the traditional LSDA+U approach of Anisimov, Liechtenstein, and co-workers (in the so-called "fully localized limit"), ${ }^{31}$ and (ii) a simplified approach of Dudarev et al. ${ }^{32}$ where only the difference $U_{\text {eff }}$ $=U-J$ enters. As shown in the Appendix the latter approach (ii) is identical to approach (i) when $J=0$, so that the difference between these two approaches can be discussed in terms of a $J$ dependence. As pointed out above, the structural and ferroelectric properties do not depend strongly on the precise values of $U$ and $J$ and are therefore basically identical for both approaches. In contrast, the magnetization of $\mathrm{BiFeO}_{3}$, which is due to a small canting of the mainly antiferromagnetically coupled magnetic moments of the $\mathrm{Fe}$ cations, ${ }^{20}$ is strongly $J$ dependent. Although the absolute value of this canting (and therefore the macroscopic magnetization) depends strongly on $J$, the effects of strain and oxygen vacancies are basically independent of the actual value of $J$. Since the focus of the present paper is on these effects, we postpone the detailed analysis of the $J$ dependence of the magnetization to a future publication and always present two data sets for the magnetization, one obtained using $U$ $=3 \mathrm{eV}$ and $J=1 \mathrm{eV}$, the second obtained using $U=2 \mathrm{eV}$ and $J=0 \mathrm{eV}$ [or equivalently approach (ii) with $U_{\text {eff }}=2 \mathrm{eV}$ ]. Our conclusions regarding the effects of strain and oxygen vacancies on the magnetization in $\mathrm{BiFeO}_{3}$ apply independently to both data sets. We point out that a significant $J$ dependence is only observed for the canting of the local magnetic moments of the Fe cations. The absolute values of these magnetic moments are rather independent of $J$, as long as $J$ is varied within reasonable limits $(J<1.5 \mathrm{eV})$.

For the calculation of the ferroelectric polarization we use the Berry-phase approach developed by Vanderbilt and King-Smith. ${ }^{33-35}$ In this theory the polarization of a periodic solid is represented by a three-dimensional lattice, and experimentally accessible polarization differences are obtained by connecting two points of the "polarization lattices" of the initial and final states, which can be transformed into each other through a continuous "path" of insulating intermediate states. In the present case special care must be taken in determining which points of the initial and final state polarization lattices have to be connected. First, because the value of the polarization difference in $\mathrm{BiFeO}_{3}$ is comparable to the distance between neighboring points on the polarization lattices, ${ }^{22}$ and in addition, because the direction of the spontaneous polarization in the monoclinically strained structures and in the supercells containing oxygen vacancies is not symmetry restricted. In such cases the noncentrosymmetric distortions of the ionic positions in the corresponding systems are gradually reduced until an unambiguous connection can be made, i.e., the polarization of some intermediate states is explicitly calculated.

\section{RESULTS AND DISCUSSION}

\section{A. Strain dependence of the electric polarization for (111) oriented films}

We first investigate the effect of strain corresponding to a (111) orientation of the substrate. This geometry preserves the rhombohedral symmetry found in the unstrained system 
TABLE I. Strain $\epsilon$, in-plane lattice parameter $a_{\text {hex }}$, relaxed out-of-plane lattice parameter $c_{\text {hex }}^{(0)}$, volume $V$ of the rhombohedral unit cell (containing two formula units), absolute and relative displacements of $\mathrm{Fe}$ and $\mathrm{O}$ ions along the [111] direction \{compared to an ideal centrosymmetric reference structure with the same lattice parameters, $\left.u_{i}(\epsilon)=R_{i}(\epsilon)-R_{i, 0}(\epsilon), \Delta u_{i}(\epsilon)=\left[u_{i}(\epsilon) / u_{i}(0)\right]-1\right\}$, spontaneous polarization $P_{s}$, and magnetization $M_{s}$ for $\mathrm{BiFeO}_{3}$ strained within the (111) plane. Upper values are obtained using the LSDA, lower values are obtained using the LSDA+U method. For the LSDA+U magnetization, the first value refers to $U=2 \mathrm{eV}, J=0 \mathrm{eV}$ and the second value refers to $U=3 \mathrm{eV}, J=1 \mathrm{eV}$ (see Sec. II for details).

\begin{tabular}{cccccccccc}
\hline \hline$\epsilon(\%)$ & $a_{\mathrm{hex}}(\AA)$ & $c_{\mathrm{hex}}^{(0)}(\AA)$ & $V\left(\AA^{3}\right)$ & $u_{\mathrm{Fe}}(\AA)$ & $\Delta u_{\mathrm{Fe}}(\%)$ & $u_{\mathrm{O}}(\AA)$ & $\Delta u_{\mathrm{O}}(\%)$ & $P_{s}\left(\mu \mathrm{C} / \mathrm{cm}^{2}\right)$ & $M_{s}\left(\mu_{\mathrm{B}} / \mathrm{Fe}\right)$ \\
\hline-3 & 5.326 & 13.68 & 112.01 & 0.294 & 16.8 & 0.527 & 2.5 & 102.8 \\
-1 & 5.431 & 13.45 & 114.53 & 0.268 & 6.2 & 0.520 & 1.2 & 100.1 & 0.04 \\
0 & 5.485 & 13.31 & 115.62 & 0.252 & 0 & 0.514 & 0 & 98.9 & 9.05 \\
+1 & 5.541 & 13.22 & 117.16 & 0.246 & -2.5 & 0.517 & 0.6 & 0.05 & 0.05 \\
+3 & 5.655 & 13.00 & 120.01 & 0.227 & -9.7 & 0.520 & 1.3 & 0.9 & 0.05 \\
\hline-3 & 5.343 & 13.92 & 114.71 & 0.357 & 21.8 & 0.573 & 6.2 & 07.7 & $0.02 / 0.11$ \\
0 & 5.503 & 13.48 & 117.86 & 0.293 & 0 & 0.539 & 0 & 94.0 \\
\hline \hline
\end{tabular}

and the spontaneous polarization remains oriented along the [111] direction. We consider compressive strain as well as tensile strain. We fix the nearest neighbor distance $a_{\text {hex }}$ between identical cations within the (111) plane to the values shown in Table I and then vary the out-of-plane lattice parameter $c_{\text {hex }}$ while relaxing all ionic positions. ${ }^{46}$ The spontaneous electric polarization $P_{s}$ and magnetization per Fe cation $M_{s}$ are calculated for the relaxed value of the out-ofplane parameter, $c_{\mathrm{hex}}^{(0)}$, corresponding to the minimum of the total energy with fixed in-plane parameter $a_{\text {hex }}$ (and relaxed ionic coordinates). The results are summarized in Table I. The strain is defined as $\epsilon=a_{\text {hex }} / a_{\text {hex }, 0}-1$ where $a_{\text {hex }, 0}$ is the corresponding lattice constant for the unstrained system. For comparison we mention that the lattice constant of $\mathrm{SrTiO}_{3}$, which is a commonly used substrate material, would lead to a compressive strain of $\epsilon \approx-2 \%$.

One can see that compressive epitaxial strain in the (111) plane leads to a reduction of the unit cell volume whereas tensile strain leads to a volume increase, i.e., the system does not behave like an ideal elastic medium. This result is similar to that found in Refs. 36 and 13 for $\mathrm{BaTiO}_{3}$. The spontaneous polarization $P_{s}$ of $\mathrm{BiFeO}_{3}$ increases slightly with increasing compressive strain ( $4 \%$ increase of the polarization for $\epsilon=-3 \%$ ) but the effect is much smaller than in the conventional ferroelectric systems $\mathrm{BaTiO}_{3}$ and $\mathrm{PbTiO}_{3}$. In $\mathrm{BaTiO}_{3}$ a strain of $\epsilon=-1 \%$ leads to an increase in the polarization of $\sim 35 \%$ (Ref. 36) whereas a similar strain in $\mathrm{PbTiO}_{3}$ increases the polarization by $15 \%-20 \% .{ }^{14}$ The largest strain effect on the polarization is seen in $\mathrm{SrTiO}_{3}$ which is not ferroelectric in the unstrained state but develops a spontaneous polarization in epitaxially strained films. ${ }^{7}$

We point out that the observed small change in spontaneous polarization is consistent with the small changes in ionic displacements that result from the applied strain. If we calculate the expected change in polarization based on a simple point charge model as $P_{s}(\epsilon)=P_{s}(\epsilon=0)+[1 / V(\epsilon)] \sum_{i} Z_{i}\left[R_{i}(\epsilon)\right.$ $-R_{i}(\epsilon=0)$ ] [here $Z_{i}$ is the charge associated with ion $i$ (see below), $R_{i}(\epsilon)$ is the corresponding strain-dependent ionic position, $V$ is the unit cell volume (also strain dependent), and the sum extends over all ions in the unit cell] then the resulting change in polarization for reasonable values of $Z_{i}$ is com- parable to the change found by the direct calculation of $P_{s}$ using the Berry-phase approach. Figure 1 shows the change in polarization as a function of strain, calculated with the Berry-phase approach as well as with the above formula using for $Z_{i}$ (i) the formal charges and (ii) the Born effective charges (BECs) of the unstrained structure. ${ }^{22}$ The weaker strain dependence of the spontaneous polarization in $\mathrm{BiFeO}_{3}$ compared to other ferroelectrics can therefore be traced back to the weaker strain dependence of the ionic displacements in this material.

Table I also shows the change in ionic displacements (compared to an ideal centrosymmetric reference structure with the same lattice parameters) as a function of strain. The positions of the $\mathrm{Bi}$ cations are used as reference and therefore the displacements of these ions are zero by definition. For a compressive strain of $\epsilon=-3 \%$ the displacements of the $\mathrm{Fe}$ cations change only by $\sim 17 \%$ (LSDA) whereas the displacements of the oxygen atoms are nearly strain independent. For comparison, an epitaxial strain of $-2.28 \%$ in the (001) plane of tetragonal $\mathrm{BaTiO}_{3}$ leads to a change of the displacements of the Ti cations along [001] of $\sim 52 \%$, and the displacements of the $\mathrm{O}$ anions in this case change by even more than $100 \% .^{36}$

There are three likely explanations for the weak strain dependence of the ionic displacements in $\mathrm{BiFeO}_{3}$. The first is the general high stability of ferroelectrics with high Curie

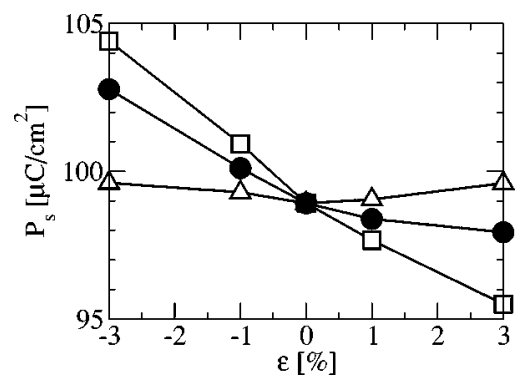

FIG. 1. Polarization $P_{s}$ as function of strain $\epsilon$ calculated within the Berry-phase approach (full circles) and by a simple point charge model (see text) using the formal charges (open triangles) and Born-effective charges of the unstrained system (open squares). 
temperatures, large ionic displacements, and large energy differences between the ground state and the centrosymmetric reference structure. $\mathrm{BiFeO}_{3}$ has a very high Curie temperature $\left(T_{C}=1123 \mathrm{~K}\right)$ and the energy gain in the ground state $R 3 c$ structure is $\sim 0.25 \mathrm{eV}$ per formula unit compared to the centrosymmetric $R \overline{3} c$ structure and $\sim 1 \mathrm{eV}$ compared to the cubic perovskite structure). This explanation is consistent with the fact that the strain dependence of the polarization is already weaker in $\mathrm{PbTiO}_{3}$ (Ref. $14, T_{C}=763 \mathrm{~K}$ ) than in $\mathrm{BaTiO}_{3}$ (Ref. 13, $T_{C}=400 \mathrm{~K}$ ). If this explanation is valid, a similar strain independence of the electric polarization should be observable for $\mathrm{LiNbO}_{3}$ which is isostructural to $\mathrm{BiFeO}_{3}$ and has a even higher Curie temperature of $1480 \mathrm{~K}$ (all Curie temperatures are taken from Ref. 37). The second possible explanation is that the mechanism driving the ferroelectric distortion in $\mathrm{BiFeO}_{3}$, namely the stereochemically active lone pair, ${ }^{38}$ is relatively inert to the changes in the lattice vectors caused by epitaxial strain. In conventional ferroelectric perovskites the ferroelectric distortion is stabilized by charge transfer from the oxygen into the unoccupied transition metal $d$ orbitals. ${ }^{39}$ This charge transfer mechanism is probably more sensitive to small changes in bond lengths than the stereochemical activity of the Bi lone electron pair. In this case the study of the strain dependence of the electric polarization in the multiferroic $\mathrm{BiMnO}_{3}$ would be of interest. The ferroelectricity in $\mathrm{BiMnO}_{3}$ is driven by the $\mathrm{Bi}$ lone pair ${ }^{38}$ but its "general stability" is not large (reported $T_{c} \sim 760 \mathrm{~K}$, Ref. 40) Thus a small (large) strain dependence in $\mathrm{LiNbO}_{3}$ and a large (small) strain dependence in $\mathrm{BiMnO}_{3}$ would confirm the general stability (lone pair) origin of the polarization stability. A third possible explanation for the weak strain dependence in $\mathrm{BiFeO}_{3}$ is the special geometry of the oxygen octahedra in the $R 3 \mathrm{c}$ structure. In ferroelectrics like $\mathrm{BaTiO}_{3}$ and $\mathrm{PbTiO}_{3}$ all ions are displaced only along the polar direction whereas in $\mathrm{BiFeO}_{3}$ (and also in $\mathrm{LiNbO}_{3}$ ) the oxygen octahedra are also rotated around this axis. The resulting geometry of the oxygen cages surrounding the transition metal cations could be less favorable for an additional straininduced displacement of the ions compared to the simpler geometry found in $\mathrm{BaTiO}_{3}$ and $\mathrm{PbTiO}_{3}$. Future studies will shed more light on this issue.

The results obtained using the LSDA+U method are very similar to the LSDA results. Although the relative change in displacements seems to be slightly larger than within the LSDA (see Table I), the relative changes in polarization are exactly the same in both cases. This reflects the fact, which has been already pointed out in Ref. 22, that the explicit treatment of electronic correlations within the LSDA+U method has only minor effect on the structural properties of this system. Apart from resulting in a slightly larger equilibrium volume, in better agreement with experimental data, the main effect is an improved description of the electronic structure resulting in a larger band gap and a stable insulating phase.

\section{B. Strain dependence of the polarization for (001) oriented films}

We now discuss the case of $\mathrm{BiFeO}_{3}$ deposited on a (001) surface. In this case the epitaxial constraint of the cubic sub-
TABLE II. Experimental lattice parameters (in $\AA$ ) found in representative $\mathrm{BiFeO}_{3}$ films (see Ref. 41) (line "Exp."), together with the corresponding lattice parameter $a_{\mathrm{rh}}$ of rhombohedral bulk $\mathrm{BiFeO}_{3}$ (Ref. 16), and the values used in the calculations for the monoclinically strained films (line "Theo."). $a$ and $b$ correspond to the two in-plane directions and $c$ to the out-of-plane direction of the monoclinic lattice. The values in the given form correspond to the lengths of the cube edges of the distorted cubic perovskite structure. Strain values $\epsilon$ are also given.

\begin{tabular}{cccccccc}
\hline \hline & & \multicolumn{3}{c}{ Thin films } & \multicolumn{3}{c}{ Thick films } \\
\cline { 3 - 8 } & $a_{\mathrm{rh}} / \sqrt{2}$ & $a / \sqrt{2}$ & $b / \sqrt{2}$ & $c$ & $a / \sqrt{2}$ & $b / \sqrt{2}$ & $c$ \\
\hline Exp. & 3.98 & 3.92 & 3.92 & 4.06 & 3.91 & 3.97 & 4.00 \\
Theo. & 3.89 & 3.83 & 3.83 & 3.97 & 3.82 & 3.88 & 3.91 \\
\hline \multicolumn{1}{c}{$\epsilon$} & $-1.5 \%$ & $-1.5 \%$ & $+2 \%$ & $-2 \%$ & $-0.2 \%$ & $+0.4 \%$ \\
\hline \hline
\end{tabular}

strate enforces $90^{\circ}$ angles of the lattice vectors within the (001) planes, in conflict with the rhombohedral distortion of bulk $\mathrm{BiFeO}_{3}$. One can expect that the competition between these two effects leads to a base-centered monoclinic structure and indeed monoclinic symmetry is found experimentally in epitaxial $\mathrm{BiFeO}_{3}$ films deposited on a (001) surface of $\mathrm{SrTiO}_{3} \cdot{ }^{41}$ The low symmetry of the monoclinic structure renders a systematic computational investigation unfeasible for this type of epitaxial strain. We have therefore performed calculations for two structures, one representative of very thin films $(\sim 100-200 \mathrm{~nm})$, the other representative of thicker films $(\sim 400 \mathrm{~nm})$, based on experimentally determined lattice parameters. ${ }^{41}$ To account for the fact that the theoretically determined lattice parameters are usually slightly different from the experimental lattice parameters we have scaled all values accordingly, so that the strain in the calculations is the same as that found experimentally. We use a monoclinic angle $\beta=89.5^{\circ}$, consistent with the experimental data. ${ }^{41}$ All lattice parameters are summarized in Table II. As reference for the theoretical lattice parameters we use the values obtained by relaxing the bulk rhombohedral unit cell using $U_{\text {eff }}=2 \mathrm{eV} .{ }^{22}$ In the thin films the large in-plane stress leads to a large out-of-plane relaxation and a $\sqrt{2} c / a$ ratio that deviates significantly from 1 . In the thick films the in-plane stress is partially released because of the two different inplane lattice parameters $a$ and $b$, leading to $\sqrt{2} c / a$ ratio (or $\sqrt{2} c / b$ ratio) closer to 1 and nearly no strain in the out-ofplane direction. The results for the electric polarization and magnetization calculated after relaxing all the ionic positions are shown in Table III. To ensure the insulating character of the systems we use the LSDA+U method for these calculations.

Due to the larger $c / a$ ratio in the "thin film" structure the direction of the polarization rotates further away from the [111] direction (towards the [001] direction) compared to the "thick film" structure. This leads to an increase in the out-ofplane component of the polarization, in spite of the fact that the effect of strain on the absolute value of the electric polarization is rather small. Thus, experiments that measure the out-of-plane component of the polarization can expect to see changes in $P$ due to this reorientation effect, even in cases 
TABLE III. Calculated absolute values of the polarization $\left|P_{s}\right|$ and out-of-plane component $\left(P_{s}\right)_{[001]}$ (in $\mu \mathrm{C} / \mathrm{cm}^{2}$ ) for the monoclinic structures. $P_{\exp }$ is the value measured in the [001] direction, (see Ref. 23) and should be compared to the calculated $\left(P_{s}\right)_{[001]}$. For the magnetization $M_{s}$, the first value refers to $U=2 \mathrm{eV}, J=0 \mathrm{eV}$ and the second value refers to $U=3 \mathrm{eV}, J=1 \mathrm{eV}$ (see Sec. II for details).

\begin{tabular}{ccccc}
\hline \hline & $\left|P_{s}\right|$ & $\left(P_{s}\right)_{[001]}$ & $P_{\exp }($ Ref. 23) & $M_{s}\left(\mu_{\mathrm{B}} / \mathrm{Fe}\right)$ \\
\hline Thin films & 94.8 & 63.4 & $50-60$ & $0.03 / 0.10$ \\
Thick films & 92.1 & 57.0 & $25-30$ & $0.03 / 0.11$ \\
\hline \hline
\end{tabular}

where the strain dependence of the magnitude of the polarization is small.

Comparing the calculated values of the spontaneous polarization with the experimental data for (001) oriented films of different thickness from Ref. 23 (see Table III) shows that the theoretical values agree reasonably well with the experimental data for the thin films (perpendicular component of the polarization). The reported experimental value for the thicker films seems to be smaller than the calculated value. This could be due to incomplete switching of the polarization in the thicker films.

In summary, the strong dependency of the polarization from the film thickness reported in Ref. 23 is probably a sum of two effects: (i) the rotation of the polarization away from the [111] direction in the thinner films due to the increased $c / a$ ratio, and (ii) incomplete switching of the polarization in the thicker films.

\section{Strain dependence of the magnetization}

It can be seen from Tables I and III that there is no significant strain dependence of the magnetization in $\mathrm{BiFeO}_{3}$. The absolute value of the magnetization (resulting from the canting of the $\mathrm{Fe}$ magnetic moments) is significantly larger for the traditional $J$ dependent LSDA $+\mathrm{U}$ treatment compared to the simplified approach corresponding to $J$ $=0 \mathrm{eV}$ (see Sec. II) but in both cases this value is not significantly changed by strain, either for the rhombohedral symmetry (Table I) or for the monoclinically strained structure (Table III).

\section{Influence of oxygen vacancies on the electric and magnetic properties}

To investigate the influence of oxygen vacancies on the ferroelectric and magnetic properties of $\mathrm{BiFeO}_{3}$ we perform calculations for a unit cell doubled along one of the rhombohedral lattice vectors (so that it contains four formula units), in which we remove one oxygen atom and then relax all ionic positions in the supercell. We then calculate total and partial densities of states, electric polarization, and magnetization. We do this for the rhombohedral bulk structure as well as for the thin film monoclinically distorted structure described in Sec. III B (see Table II). The use of a doubled unit cell artificially reduces the symmetry of the rhombohedral system to the monoclinic space group $B b$ (in the case of the monoclinically distorted structure this is already the
TABLE IV. Polarization $\left|P_{s}\right|$ and magnetization $M_{s}$ calculated within the LSDA+U method for the systems with oxygen vacancies. The upper (middle) three lines correspond to the three vacancy configurations based on the rhombohedral bulk structure (monoclinic thin film structure). The last line corresponds to the tripled unit cell. For the magnetization, the first value refers to $U=2 \mathrm{eV}$, $J=0 \mathrm{eV}$ and the second value refers to $U=3 \mathrm{eV}, J=1 \mathrm{eV}$ (see Sec. II for details).

\begin{tabular}{ccc}
\hline \hline & $\left|P_{s}\right|\left(\mu \mathrm{C} / \mathrm{cm}^{2}\right)$ & $M_{s}\left(\mu_{\mathrm{B}} / \mathrm{Fe}\right)$ \\
\hline I & 96.6 & $0.01 / 0.07$ \\
II & 95.0 & $0.07 / 0.14$ \\
III & 97.1 & $0.05 / 0.11$ \\
\hline I & 97.1 & $0.02 / 0.08$ \\
II & 94.6 & $0.07 / 0.14$ \\
III & 99.9 & $0.05 / 0.10$ \\
\hline tripled & 96.5 & $0.06 / 0.12$ \\
\hline \hline
\end{tabular}

space group of the original unit cell). In this lowered symmetry there are three inequivalent groups of oxygen anions. Removing an oxygen anion from one of the three groups in turn leads to three different arrangements of oxygen vacancies. In the following we refer to these different configurations (in a somewhat arbitrary way) as configurations I, II, and III. The corresponding supercells have a vacancy concentration of $8.3 \%$, which means that one out of 12 oxygen anions is missing. This can formally be written as $\mathrm{BiFeO}_{3-\delta}$ with $\delta=0.25$.

All systems are metallic within the LSDA but become insulating within the LSDA+U. Table IV shows the values for the electric polarization and magnetization for all supercells calculated within the LSDA $+\mathrm{U}$ method. Remarkably, the electric polarization is not significantly affected by the presence of oxygen vacancies and the accompanying structural and electronic distortions. This again reflects the high stability of the ferroelectric configuration in this system. Even the combined presence of epitaxial strain and oxygen vacancies in the monoclinically distorted structures does not lead to significant changes in the ferroelectric polarization $P_{s}$.

Figure 2 shows the densities of states (total and partial Fe d) for vacancy configuration I based on the rhombohedral bulk structure together with the corresponding densities of states for the same structure without oxygen vacancies. The densities of states for the other systems all look very similar to that shown in Fig. 2. The four Fe cations in the supercell containing the vacancy can be divided in two classes which we call " $\mathrm{Fe}^{3+}$ " and " $\mathrm{Fe}^{2+}$." The partial $d$ densities of states for the two $\mathrm{Fe}^{3+}$ cations are very similar to the case without the oxygen vacancies: the (local) majority spin states are completely filled and the (local) minority spin states are completely empty (apart from a small contribution arising from the hybridization with the $\mathrm{O} 2 p$ states), indicating a $d^{5}$ high-spin electronic configuration. The unoccupied minority $d$ states are split into the $t_{2 g}$ and $e_{g}$ manifolds characteristic of the predominantly octahedral symmetry of the crystal field. The partial densities of states for the $\mathrm{Fe}^{2+}$ cations are 


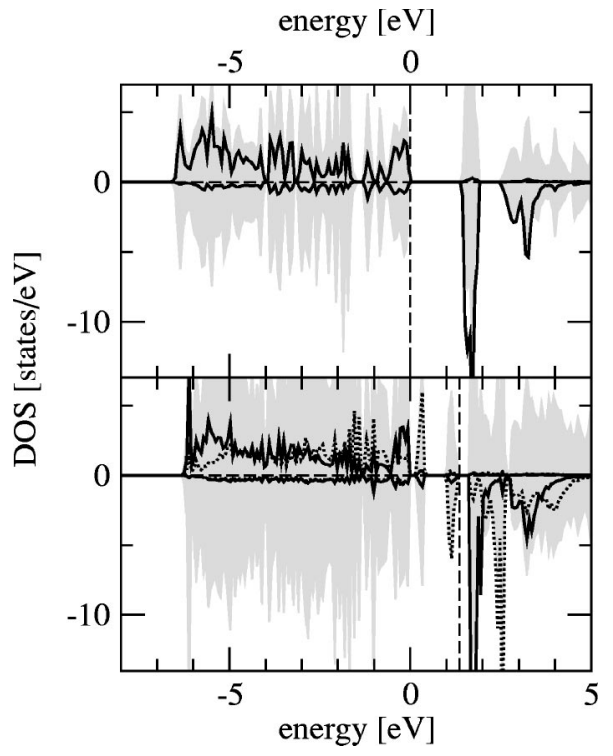

FIG. 2. Total (gray shaded) and partial Fe $d$ densities of states for the unstrained rhombohedral structure (upper panel) and for vacancy configuration I (lower panel) calculated for $U_{\text {eff }}=2 \mathrm{eV}$. Minority spin states are shown with a negative sign. The full lines correspond to the $\mathrm{Fe}^{3+}$ ions, the dotted lines in the lower panel correspond to the $\mathrm{Fe}^{2+}$ ions. The dashed vertical lines indicate the highest occupied energy levels. Zero energy is set to the upper edge of the $\mathrm{Fe}^{3+}$ majority spin bands.

significantly different from this. Here, the $t_{2 g}$ minority states are partially filled by approximately one electron (indicating a high spin $d^{6}$ electron configuration) and there is a small gap between the occupied and unoccupied minority $t_{2 g}$ states. The densities of states therefore suggest a picture of distinct $\mathrm{Fe}^{2+}$ and $\mathrm{Fe}^{3+}$ cations with $d^{5}$ and $d^{6}$ electron configurations, respectively.

Although the presence of both $\mathrm{Fe}^{2+}$ and $\mathrm{Fe}^{3+}$ seems rather obvious from the analysis of the densities of states, the local charges, obtained by integrating the partial densities of states up to the Fermi energy, differ only slightly $(\sim 0.1 e)$ for the two types of Fe cations. Furthermore, these charges do not represent the formal charges corresponding to the electron configurations mentioned in the previous paragraph. The problem of assigning local (static) charges based on the continuous electron density in periodic solids is well known (see, e.g., Ref. 42). In practice, local quantities are found by defining spheres around the atomic sites and projecting the Bloch functions onto a local basis. Since the radii of such spheres are arbitrary within reasonable limits, and the intermediate region between these spheres is either neglected or double counted, the local quantities defined in this way are not uniquely defined. Furthermore, due to the formation of band states within a periodic solid, the angular momentum $l$ is no longer a good quantum number. Even a pure " $d$ band," constructed as the Bloch-sum of localized $d$ orbitals, usually contains some $s$ and $p$ character when projected onto a local $l$ basis. It is therefore clear that locally defined charges are poor indicators for the oxidation states of the ions. The clear qualitative differences in the local densities of states appear to be much more appropriate for this purpose.
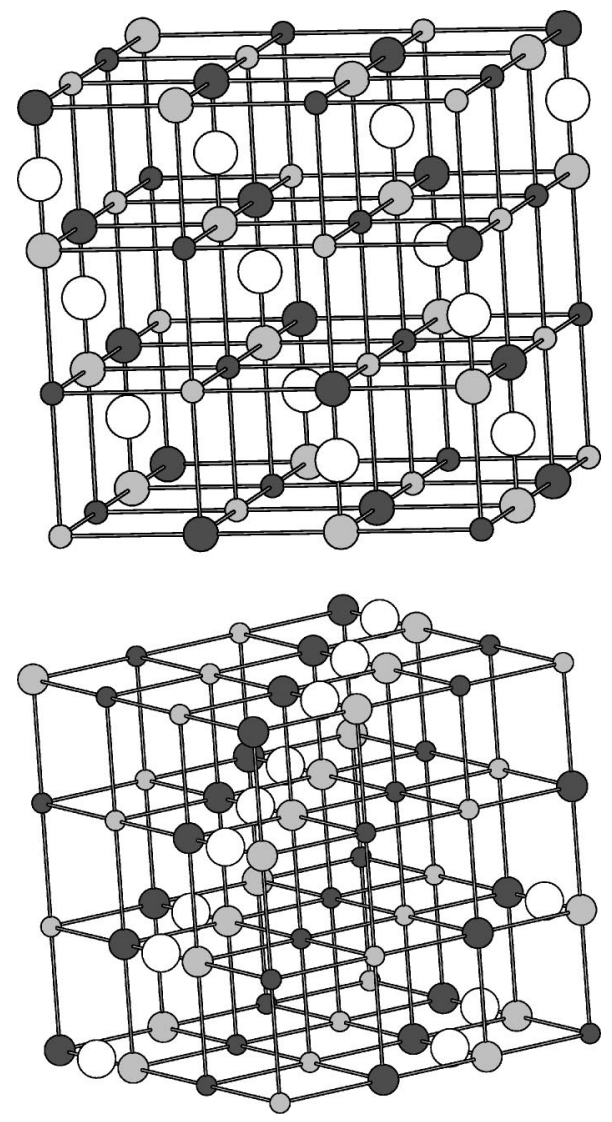

FIG. 3. Schematic picture of charge ordering in $\mathrm{BiFeO}_{2.75}$ for vacancy configuration I. Large white spheres represent oxygen vacancies. Large (small) gray spheres represent $\mathrm{Fe}^{2+}\left(\mathrm{Fe}^{3+}\right)$ ions. Different shadings indicate the two different antiferromagnetic spin orientations. For simplicity all ions are placed at the corresponding ideal positions within the cubic perovskite structure. On the left side stripes of $\mathrm{Fe}^{2+}$ ions along the [110] direction can be identified. On the right side the quasiplanar arrangement of the oxygen vacancies is visible.

The three-dimensional arrangement of $\mathrm{Fe}^{2+}$ and $\mathrm{Fe}^{3+}$ is shown schematically in Fig. 3 for vacancy configuration I. The $\mathrm{Fe}^{2+}$ cations appear on the sites adjacent to the oxygen vacancy. The quasiplanar arrangement of oxygen vacancies shown in Fig. 3 is a result of the particular restricted supercell geometry. A more isotropic distribution of oxygen vacancies and a smaller (and probably more realistic) vacancy concentration would require the use of larger supercells.

To validate our results also for a slightly smaller vacancy concentration we repeated our calculations for a tripled unit cell containing six formula units of $\mathrm{BiFeO}_{3-\delta}$, corresponding to a vacancy concentration of $5.6 \%$ or $\delta \approx 0.17$. In this case we describe the rhombohedral lattice using hexagonal lattice vectors where one unit cell of the hexagonal lattice contains three lattice points of the rhombohedral lattice. The resulting hexagonal unit cell corresponds to space group $P 3$ and in this case there are six groups of inequivalent oxygen anions. To reduce the computational effort, we only treat one possible arrangement of oxygen vacancies for the tripled unit cell, which is obtained by arbitrarily removing one of the oxygen anions. This calculation is done only for the rhom- 
bohedral bulk lattice parameters and using the LSDA $+\mathrm{U}$ method.

The calculated polarization for the tripled unit cell with oxygen vacancy is shown in Table IV. Also in this case the polarization is not significantly affected by the presence of the oxygen vacancies. Similar to the doubled unit cells, we obtain two distinct classes of $\mathrm{Fe}$ cations that can be interpreted as $\mathrm{Fe}^{2+}$ and $\mathrm{Fe}^{3+}$, with local $\mathrm{Fe} d$ densities of states very similar to those shown in Fig. 2. In this case the ratio of $\mathrm{Fe}^{2+}$ to $\mathrm{Fe}^{3+}$ is $1: 2$, as required by the charge neutrality of the system. Again, the $\mathrm{Fe}^{2+}$ cations appear on the sites adjacent to the oxygen vacancy. Therefore, although our supercells enforce a rather artificial ordered arrangement of oxygen vacancies and the corresponding vacancy concentrations are relatively high, we conclude that the incorporation of oxygen vacancies in $\mathrm{BiFeO}_{3}$ leads to the formation of $\mathrm{Fe}^{2+}$ on the sites adjacent to the vacancy.

We now turn our attention to the effect of vacancies on the macroscopic magnetization which is also shown in Table IV. Since the pairs of $\mathrm{Fe}^{2+}$ cations are always situated on neighboring positions of the magnetic lattice, and are therefore antiferromagnetically aligned, no net magnetization results from a ferrimagnetic arrangement of $\mathrm{Fe}^{2+}$ and $\mathrm{Fe}^{3+}$ cations as occurs, for example, in magnetite. ${ }^{43}$ The magnetization is therefore still caused entirely by the small canting of the mainly antiferromagnetically oriented magnetic moments of the Fe cations, as shown in Ref. 20 (weak ferromagnetism). ${ }^{19}$ Two observations can be made by inspection of Table IV. First, there is no significant difference between the strained and unstrained systems. Second, in some cases the magnetization is enhanced compared to the bulk value, whereas in other cases it is decreased. The differences are slightly more pronounced for the data set corresponding to $U=2 \mathrm{eV}, J=0 \mathrm{eV}$ than for the data set corresponding to $U=3 \mathrm{eV}, J=1 \mathrm{eV}$, but no clear trends can be identified. The observed changes in magnetization for the various vacancy configurations cannot be understood by considering simple changes in coordination between the magnetically coupled cations (which are the same for all vacancy configurations) but rather depend on the details of the structural relaxation of both cations and anions. This is not surprising since the Dzyaloshinskii-Moriya interaction, which is responsible for the canting of the magnetic moments, ${ }^{19}$ is closely related to the superexchange interaction, which in turn is known to be very sensitive to small structural changes. ${ }^{44}$ The observed changes in magnetization caused by the presence of oxygen vacancies cannot explain the strong increase in magnetization reported for the thin films of $\mathrm{BiFeO}_{3} \cdot{ }^{23}$

\section{SUMMARY AND CONCLUSIONS}

In summary, our calculations show that the ferroelectric polarization in multiferroic $\mathrm{BiFeO}_{3}$ is extremely insensitive to both strain and the presence of oxygen vacancies. This insensitivity of the polarization is due to a corresponding insensitivity of the ionic displacements and is in striking contrast to what has been found for most conventional perovskite ferroelectrics. At present it is not clear if this stability is due to a general high stability of the ferroelectric state in
$\mathrm{BiFeO}_{3}$, reflected also in its large ionic displacements and high Curie temperature $\left(T_{C}=1123 \mathrm{~K}\right),{ }^{37}$ if this stability is a special feature of the different mechanism driving the ferroelectric distortion in this class of Bi-containing multiferroic materials, namely the stereochemically active Bi lone electron pair, ${ }^{38}$ or if it is due to the special geometry of the $R 3 \mathrm{c}$ space group containing oxygen octahedra rotations in addition to the polar displacements. Future studies of the strain dependence of the ferroelectric polarization in the high $T_{C}$ ferroelectric $\mathrm{LiNbO}_{3}$ and the lone-pair active multiferroic $\mathrm{BiMnO}_{3}$ will help to solve this issue.

The incorporation of oxygen vacancies in $\mathrm{BiFeO}_{3}$ leads to the formation of $\mathrm{Fe}^{2+}$ which can be identified by the clear qualitative differences in the local densities of states, but the actual charge disproportionation is small. The presence of oxygen vacancies can affect the value of the macroscopic magnetization, although the observed changes are too small to explain the strong increase of the magnetization reported for thin $\mathrm{BiFeO}_{3}$ film. ${ }^{23}$ These effects of oxygen vacancies are independent of the strain state of the system.

Recently, measurements of the electric polarization for (001), (101), and (111) oriented films of $\mathrm{BiFeO}_{3}(200 \mathrm{~nm}$ thickness) were reported ${ }^{24}$ and the experimental data could be explained as resulting from different projections of the same polarization vector. This supports the notion that both magnitude and orientation of the polarization in $\mathrm{BiFeO}_{3}$ are not significantly affected by strain, since the strain tensor differs considerably for different film orientations. The reported value of $\sim 100 \mu \mathrm{C} / \mathrm{cm}^{2}$ for the (111) oriented film ${ }^{24}$ agrees well with the polarization values calculated in this work.

\section{ACKNOWLEDGMENTS}

The authors thank R. Ramesh, C. J. Fennie, and K. M. Rabe for valuable discussions and $\mathrm{R}$. Ramesh for providing the structural data for the (001) oriented films. This work was supported by the MRSEC program of the National Science Foundation under Award No. DMR00-80034.

\section{APPENDIX: EQUIVALENCE OF THE TWO LSDA+U APPROACHES FOR $J=0$}

In the LSDA+U approach of Dudarev et al. ${ }^{32}$ the total energy of the system is expressed as follows [Eq. (5) of Ref. 32 , generalized for noncollinear spin systems]:

$$
E=E_{\mathrm{LSDA}}+\frac{U_{\mathrm{eff}}}{2}\left(n-\sum_{m, m^{\prime}, s, s^{\prime}} n_{m m^{\prime}}^{s s^{\prime}} n_{m^{\prime} m}^{s^{\prime} s}\right) .
$$

Here, $n_{m m^{\prime}}^{s s^{\prime}}$ are the elements of the orbital density matrix and $n=\Sigma_{s, m} n_{m m}^{s s}$ is the total number of $d$ electrons at the corresponding ion. Summation over all sites containing $d$ electrons has been suppressed for simplicity.

The corresponding expression for the LSDA $+\mathrm{U}$ approach of Anisimov, Liechtenstein, and coworkers is ${ }^{31}$ 


$$
\begin{aligned}
E= & E_{\mathrm{LSDA}}+\frac{1}{2} \sum_{\{m, s\}}\left\{\left\langle m_{1} m_{3}\left|V_{\mathrm{ee}}\right| m_{2} m_{4}\right\rangle n_{m_{1} m_{2}}^{s_{1} s_{1}} n_{m_{3} m_{4}}^{s_{2} s_{2}}\right. \\
& \left.-\left\langle m_{1} m_{3}\left|V_{\mathrm{ee}}\right| m_{4} m_{3}\right\rangle n_{m_{1} m_{2}}^{s_{1} s_{2}} n_{m_{3} m_{4}}^{s_{2} s_{1}}\right\} \\
& -\frac{U}{2} n(n-1)+\frac{J}{2} \sum_{s} n^{s}\left(n^{s}-1\right) .
\end{aligned}
$$

Here, $n^{s}=\Sigma_{m} n_{m m}^{s s}$ is the total number of $d$ electrons with spin $s$ and $\left\langle m_{1} m_{3}\left|V_{\text {ee }}\right| m_{2} m_{4}\right\rangle$ are the matrix elements of the screened electron-electron interaction. These matrix elements are defined in terms of Slater-integrals $F^{k}$ as

$$
\left\langle m_{1} m_{3}\left|V_{\mathrm{ee}}\right| m_{2} m_{4}\right\rangle=\sum_{k} a_{k}\left(m_{1}, m_{3}, m_{2}, m_{4}\right) F^{k}
$$

with

$$
a_{k}\left(m_{1}, m_{3}, m_{2}, m_{4}\right)=\frac{4 \pi}{2 k+1} \sum_{q=-k}^{k}\left\langle l m_{1}\left|Y_{k q}\right| l m_{2}\right\rangle\left\langle l m_{3}\left|Y_{k q}^{*}\right| l m_{4}\right\rangle .
$$

The Slater integrals are related to the parameters $U$ and $J$ via $U=F^{0}, \quad J=\left(F^{2}+F^{4}\right) / 14$, where one usually sets $F^{2} / F^{4}$ $=0.625$. For $J=0$ the only nonvanishing term in the sum of Eq. (A3) is for $k=0$, and one can see from Eq. (A4) that $a_{0}\left(m_{1}, m_{2}, m_{3}, m_{4}\right)=\delta_{m_{1} m_{2}} \delta_{m_{3} m_{4}}$. For $J=0$ the matrix elements (A3) are therefore simply given by

$$
\left\langle m_{1} m_{3}\left|V_{\text {ee }}\right| m_{2} m_{4}\right\rangle=U \delta_{m_{1} m_{2}} \delta_{m_{3} m_{4}} .
$$

Using (A5) in (A2) one obtains

$$
E=E_{\mathrm{LSDA}}+\frac{U}{2}\left(n-\sum_{m, m^{\prime}, s, s^{\prime}} n_{m m^{\prime}}^{s s^{\prime}} n_{m^{\prime} m}^{s^{\prime} s}\right)
$$

which is just Eq. (A1) with $U_{\text {eff }}=U$. The LSDA $+U$ approach of Dudarev et al. ${ }^{32}$ is therefore included in the approach of Ref. 31 as the special case $J=0$.
*Electronic address: ederer@mrl.ucsb.edu

${ }^{1}$ M. Fiebig, T. Lottermoser, D. Fröhlich, A. V. Goltsev, and R. V. Pisarev, Nature (London) 419, 818 (2002).

${ }^{2}$ T. Kimura, T. Goto, H. Shintani, K. Ishizaka, T. Arima, and Y. Tokura, Nature (London) 426, 55 (2003).

${ }^{3}$ N. Hur, S. Park, P. A. Sharma, J. S. Ahn, S. Guha, and S.-W. Cheong, Science 429, 392 (2004).

${ }^{4}$ T. Lottermoser, T. Lonkai, U. Amann, D. Hohlwein, J. Ihringer, and M. Fiebig, Nature (London) 430, 541 (2004).

${ }^{5}$ G. A. Smolenskii and I. E. Chupis, Sov. Phys. Usp. 25, 475 (1982).

${ }^{6}$ K. J. Choi, M. Biegalski, Y. L. Li, A. Sharan, J. Schubert, R. Uecker, P. Reiche, Y. B. Chen, X. Q. Pan, V. Gopalan, L-Q. Chen, D. G. Schlom, and C. B. Eom, Science 306, 1005 (2004).

${ }^{7}$ J. H. Haeni, P. Irvin, W. Chang, R. Uecker, P. Reiche, Y. L. Li, S. Choudhury, W. Tian, M. E. Hawley, B. Craigo, A. K. Tagantsev, X. Q. Pan, S. K. Streiffer, L. Q. Chen, S. W. Kirchoefer, J. Levy, and D. G. Schlom, Nature (London) 430, 758 (2004).

${ }^{8}$ N. A. Hill, Annu. Rev. Mater. Res. 32, 1 (2002).

${ }^{9}$ B. B. van Aken, T. T. M. Palstra, A. Filippetti, and N. A. Spaldin, Nat. Mater. 3, 164 (2004).

${ }^{10}$ F. Tsui, M. C. Smoak, T. K. Nath, and C. B. Eom, Appl. Phys. Lett. 76, 2421 (2000).

${ }^{11}$ Q. Gan, R. A. Rao, C. B. Eom, J. L. Garrett, and M. Lee, Appl. Phys. Lett. 72, 978 (1998).

${ }^{12}$ R. O. Jones and O. Gunnarsson, Rev. Mod. Phys. 61, 689 (1989).

${ }^{13}$ J. B. Neaton and K. M. Rabe, Appl. Phys. Lett. 82, 1586 (2003).

${ }^{14}$ C. Bungaro and K. M. Rabe, Phys. Rev. B 69, 184101 (2004).

${ }^{15}$ O. Diéguez, S. Tinte, A. Antons, C. Bungaro, J. B. Neaton, K. M. Rabe, and D. Vanderbilt, Phys. Rev. B 69, 212101 (2004).

${ }^{16}$ F. Kubel and H. Schmid, Acta Crystallogr., Sect. B: Struct. Sci. 46, 698 (1990).

${ }^{17}$ P. Fischer, M. Polemska, I. Sosnowska, and M. Szymański, J. Phys. C 13, 1931 (1980).
${ }^{18}$ I. Sosnowska, T. Peterlin-Neumaier, and E. Streichele, J. Phys. C 15, 4835 (1982).

${ }^{19}$ T. Moriya, Phys. Rev. 120, 91 (1960).

${ }^{20}$ C. Ederer and N. A. Spaldin, Phys. Rev. B 71, 060401 (2005).

${ }^{21}$ J. R. Teague, R. Gerson, and W. J. James, Solid State Commun. 8, 1073 (1970).

${ }^{22}$ J. B. Neaton, C. Ederer, U. V. Waghmare, N. A. Spaldin, and K. M. Rabe, Phys. Rev. B 71, 014113 (2005).

${ }^{23}$ J. Wang, J. B. Neaton, H. Zheng, V. Nagarajan, S. B. Ogale, B. Liu, D. Viehland, V. Vaithyanathan, D. G. Schlom, U. V. Waghmare, N. A. Spaldin, K. M. Rabe, M. Wuttig, and R. Ramesh, Science 299, 1719 (2003).

${ }^{24}$ J. Li, J. Wang, M. Wuttig, R. Ramesh, N. Wang, B. Ruette, A. P. Pyatakov, A. K. Zvezdin, and D. Viehland, Appl. Phys. Lett. 84, 5261 (2004).

${ }^{25}$ F. Bai, J. Wang, M. Wuttig, J. Li, N. Wang, A. P. Pyatakov, A. K. Zvezdin, L. E. Cross, and D. Viehland, Appl. Phys. Lett. 86, 032511 (2005).

${ }^{26}$ P. E. Blöchl, Phys. Rev. B 50, 17953 (1994).

${ }^{27}$ G. Kresse and J. Furthmüller, Phys. Rev. B 54, 11169 (1996).

${ }^{28}$ G. Kresse and D. Joubert, Phys. Rev. B 59, 1758 (1999).

${ }^{29}$ H. J. Monkhorst and J. D. Pack, Phys. Rev. B 13, 5188 (1976).

${ }^{30}$ P. E. Blöchl, O. Jepsen, and O. K. Andersen, Phys. Rev. B 49, 16223 (1994).

${ }^{31}$ V. I. Anisimov, F. Aryasetiawan, and A. I. Liechtenstein, J. Phys.: Condens. Matter 9, 767 (1997).

${ }^{32}$ S. L. Dudarev, G. A. Botton, S. Y. Savrasov, C. J. Humphreys, and A. P. Sutton, Phys. Rev. B 57, 1505 (1998).

${ }^{33}$ R. D. King-Smith and D. Vanderbilt, Phys. Rev. B 47, R1651 (1993).

${ }^{34}$ D. Vanderbilt and R. D. King-Smith, Phys. Rev. B 48, 4442 (1994).

${ }^{35}$ R. Resta, Rev. Mod. Phys. 66, 899 (1994).

${ }^{36}$ J. B. Neaton, C.-L. Hsueh, and K. M. Rabe, cond-mat/0204511 (unpublished). 
${ }^{37}$ Landolt-Börnstein-Group III Condensed Matter (Springer Verlag Heidelberg, 2002) Vol. 36A.

${ }^{38}$ R. Seshadri and N. A. Hill, Chem. Mater. 13, 2892 (2001).

${ }^{39}$ R. E. Cohen, J. Phys. Chem. Solids 61, 139 (2000).

${ }^{40}$ T. Kimura, S. Kawamoto, I. Yamada, M. Azuma, M. Takano, and Y. Tokura, Phys. Rev. B 67, 180401(R) (2003).

${ }^{41} \mathrm{R}$. Ramesh (private communication).

${ }^{42}$ P. Ghosez, J.-P. Michenaud, and X. Gonze, Phys. Rev. B 58, 6224 (1998).

${ }^{43}$ C. G. Shull, E. O. Wollan, and W. A. Strauser, Phys. Rev. 81, 483
(1951).

${ }^{44} \mathrm{P}$. W. Anderson, in Magnetism, edited by G. T. Rado and H. Suhl (Academic Press, New York 1963), Vol. 1, Chap. 2, pp. 25-83.

${ }^{45}$ International Tables of Crystallography, Volume A: Space Group Symmetry, edited by T. Hahn (Kluwer, Dordrecht 2002).

${ }^{46}$ The rhombohedral lattice can be described either using a rhombohedral or a hexagonal setup (see, e.g., Ref. 45). We use one or the other notation, depending on what is appropriate in the given context. 